\title{
Compactified Jacobians and Torelli Map ${ }^{\dagger}$
}

By

\author{
Valery ALexeEv*
}

\begin{abstract}
We compare several constructions of compactified jacobians - using semistable sheaves, semistable projective curves, degenerations of abelian varieties, and combinatorics of cell decompositions - and show that they are equivalent. We give a detailed description of the "canonical compactified jacobian" in degree $g-1$. Finally, we explain how Kapranov's compactification of configuration spaces can be understood as a toric analog of the extended Torelli map.
\end{abstract}

\section{Introduction}

There are many papers devoted to compactifying (generalized) jacobians of curves and families of curves. Some of them are concerned primarily with existence, some provide a finer description. The approaches vary widely: some constructions use moduli of semistable rank-1 sheaves, some use semistable projective curves, some use combinatorics of cell decompositions; yet others use degenerations of principally polarized abelian varieties and various notions of stable varieties.

One aim of this survey is to give a definitive account in the case of nodal curves and to show, pleasingly, that in this case all of the known approaches are equivalent and produce isomorphic varieties, with the degeneration of PPAVs approach being the special case of degree $g-1$. Combining the known results we then describe these varieties in detail.

The degree $g-1$ case deserves a special attention since in this case the compactified Jacobian is unique and comes with a canonical theta divisor. It is also intimately connected with the Torelli map.

Communicated by S. Mori. Received August 2, 2004.

2000 Mathematics Subject Classification(s): 14D22

${ }^{\dagger}$ This article is an invited contribution to a special issue of Publications of RIMS commemorating the fortieth anniversary of the founding of the Research Institute for Mathematical Sciences.

*Department of Mathematics University of Georgia Athens, GA 30602.

e-mail: valery@math.uga.edu

(C) 2004 Research Institute for Mathematical Sciences, Kyoto University. All rights reserved. 
Recall that the classical Torelli map $\mathfrak{t}: \mathrm{M}_{g} \rightarrow \mathrm{A}_{g}$ is a morphism from the moduli space of smooth genus $g$ curves to the moduli space of principally polarized abelian varieties which associates to a smooth curve $X$ of genus $g$ its Jacobian: a principally polarized abelian variety $\operatorname{Pic}^{0} X$, or - as we prefer to think - a stable pair $\left(\mathrm{Pic}^{g-1} X, \Theta_{g-1}\right)$ with the theta divisor.

As documented by Namikawa in [Nam76], it was Mumford who first noted the fact that $\mathfrak{t}$ extends to a regular morphism $\overline{\mathfrak{t}}-$ which we will call the toroidal Torelli map - from the Deligne-Mumford compactification $\overline{\mathrm{M}}_{g}$ to the toroidal compactification $\overline{\mathrm{A}}_{g}^{\mathrm{Vor}}$ for the 2nd Voronoi fan. The proof of this fact consists of a purely combinatorial argument about fans. The extension property of $\overline{\mathrm{A}}_{g}^{\text {Vor }}$ was the impetus for the study of this compactification carried out by Namikawa and for his pioneering theory of stable quasiabelian varieties.

On the other hand, in [Ale02] we showed that $\overline{\mathrm{A}}_{g}^{\text {Vor }}$ has a functorial meaning of the main irreducible component in the moduli space of stable pairs $(P, D)$, where $P$ is a reduced projective variety with semiabelian group action and $D$ a divisor on it, satisfying a few natural conditions. Hence, the toroidal Torelli map is a morphism from one moduli space of stable objects, lower-dimensional but nonlinear, to another, higher-dimensional but linear; and functorially it is defined by associating to a stable curve $X$ the moduli space $\operatorname{Jac}_{g-1} X$ of semistable rank-1 "torsion-free" sheaves of degree $g-1$ on $X$ together with the natural theta divisor $\Theta_{g-1}$.

The second part of this survey concerns Kapranov's compactification of configuration spaces [Kap93] (and a related work of Lafforgue on the compactification of thin Schubert cells [Laf03]). We show that Kapranov's construction can be understood as an application of a toric analog of the extended Torelli map $\overline{\mathfrak{t}}$. More precisely, each configuration space $\mathrm{KS}_{r, n}$ of [Kap93] comes with morphism, a toric analog of Torelli map, to an appropriate analog of $\overline{\mathrm{A}}_{g}^{\text {Vor }}$, and the compactification $\overline{\mathrm{KS}}_{r, n}$ constructed coincides with the closure of the image. As in the toroidal case, both the source $\overline{\mathrm{KS}}_{r, n}$ and the target space can be interpreted as moduli spaces of stable pairs. Moreover, the combinatorics involved is to a remarkable degree similar to the toroidal case, with periodic polyhedral tilings replaced by tilings of a single polytope.

When working with individual varieties, we assume them to be defined over an algebraically closed field $k$ of arbitrary characteristic. When working with families, we work in the category of locally Noetherian schemes over a field or a Dedekind domain. 


\section{$\S 1$. Compactified Jacobians of Nodal Curves}

1.1. Construction of moduli spaces of semistable sheaves is a basic application of Geometric Invariant Theory. For sheaves on curves the main reference is Seshadri [Ses82], for a narrower case of irreducible curves (where the moduli space is fine) the references are works [D'S79, AIK77, AK80, AK79] of D'Souza, Altman, Iarrobino, Kleiman and Altman. For another special case, of nodal but possibly reducible curves, it is Oda-Seshadri [OS79]. For a more general case, of semistable coherent sheaves on arbitrary projective families, see [Sim94]. Let us recall the basic definitions and results.

Let $X$ be a projective scheme over an algebraically closed field and $L$ be a polarization, i.e. an ample invertible sheaf on $X$. For any coherent sheaf $F$ on $X$ one defines its Hilbert polynomial

$$
p(F, n)=\chi\left(X, F \otimes L^{n}\right)=r n^{d} / d !+a n^{d-1} /(d-1) !+\cdots
$$

The integer $r=r(F)$ is called the rank of $F$. The ratio $\mu(F)=a / r$ is called the slope of $F$ and

$$
p(F, n) / r(F)=n^{d} / d !+\mu(F) n^{d-1} /(d-1) !+\cdots
$$

is the normalized Hilbert polynomial.

A sheaf $F$ is called admissible (or of pure dimension, or an $S_{1}$-sheaf) if for any nonzero subsheaf $E$ of $F$ one has $d(E)=d(F)$. A sheaf $F$ is called slope-semistable (resp. slope-stable) if for any proper nonzero subsheaf $E$ of $F$ one has $\mu(E) \leq \mu(F)$ (resp. the inequality is strict). A sheaf $F$ is called $p$ semistable (resp. p-stable) if, similarly, one has the inequality $p(E, n) / r(E) \leq$ $p(F, n) / r(F)$ of normalized Hilbert polynomials for $n \gg 0$, which is equivalent to comparing the coefficients of the two polynomials lexicographically.

Every admissible sheaf $F$ has a Harder-Narasimhan filtration

$$
0=F_{0} \subset F_{1} \subset \ldots F_{s}=F
$$

such that the quotients $F_{i} / F_{i-1}$ are $p$-stable and the sequence of normalized Hilbert polynomials is decreasing. If $F$ is $p$-semistable then the sequence is constant. The graded object $\operatorname{gr}(F)=\oplus F_{i} / F_{i-1}$ does not depend on a filtration as above. Two semistable sheaves are said to be gr-equivalent if $\operatorname{gr}(F) \simeq \operatorname{gr}\left(F^{\prime}\right)$.

Now let $X \rightarrow S$ be a projective family with a relatively ample sheaf $L$. A coherent sheaf $F$ on $X$ flat over $S$ is called slope- or $p$ - (semi)stable if so is its restriction to a fiber $X_{s}$ for every geometric point $s \rightarrow S$.

Consider the functor $\mathcal{M}=\mathcal{M}_{p(n)}$ which associates to any $S$-scheme $S^{\prime}$ the set of $p$-semistable sheaves on $X^{\prime}=X \times{ }_{S} S^{\prime}$ with a fixed Hilbert polynomial $p(n)$. Then one has quite generally: 


\section{Theorem 1.2.}

(1) There exists a scheme $M$ and a natural transformation $\mathcal{M} \rightarrow \operatorname{Hom}(*, M)$ which has the universal property, i.e. for any scheme $M^{\prime}$ a natural transformation $\mathcal{M} \rightarrow \operatorname{Hom}\left(*, M^{\prime}\right)$ factors through a unique morphism $M \rightarrow M^{\prime}$.

(2) The scheme $M$ is projective over $S$.

(3) The points of every geometric fiber $M_{s}$ correspond to gr-equivalence classes of semistable sheaves on $X_{s}$.

For higher dimensions, this is [Sim94, 1.21] and the hardest part of the proof is boundedness of semistable sheaves. Simpson works over $\mathbb{C}$. However, for curves the boundedness is trivial.

The scheme $M=M_{X / S}$ is called the (coarse) moduli space of semistable sheaves. Even though it is lacking the property $\mathcal{M}(k)=M(k)$ usually asked of coarse moduli spaces, the universal property defines $M$ uniquely up to a canonical isomorphism.

1.3. Now from this very general situation let us return to the case of curves. A sheaf $F$ on a curve $X$ having support of dimension one is admissible iff it does not contain skyscraper sheaves. The normalized Hilbert polynomial

$$
p(F, n) / r(F)=n+\mu(F)
$$

has only one non-trivial coefficient $\mu(F)=\chi(F) / r(F)$, and so $p$-stability is equivalent to the slope-stability.

Suppose $X$ is a reduced curve with irreducible components $X_{i}$, the sheaf $F$ has ranks $\left(r_{i}\right)$ at generic points of $X_{i}$, and the polarization $L$ has multidegree $\left(l_{i}\right)$. Then one immediately computes:

$$
r(F)=\sum l_{i} r_{i}, \quad \mu(F)=\frac{\chi(F)}{\sum l_{i} r_{i}}
$$

Call the difference $\operatorname{deg} F:=\chi(F)-\chi\left(O_{X}\right)$ the degree of $F$. For every degree $d \in \mathbb{Z}$ and a polarization $L$, the compactified jacobian $\mathrm{Jac}_{d, L}$ is defined to be the moduli space of semistable, with respect to polarization $L$, sheaves of degree $d$ which have rank 1 at every generic point of $X$. (Note, however, that the stability condition involves checking the slope inequality for all subsheaves $E$ of $F$, and these may have rank 1 or 0 at different irreducible components of $X$.) It is a projective variety, an open and closed subscheme of the above moduli space $M_{p(n)}$ for the Hilbert polynomial $p(F, n)=n \sum l_{i}+\chi\left(O_{X}\right)+d$. 
Schemes $\mathrm{Jac}_{0, L} X$ provide natural compactifications for the ordinary Jacobian $\mathbf{P i c}^{0} X$, the group variety whose geometric points form the group $\operatorname{Pic}^{0}(X)$. For any degree $d, \mathbf{P i c}^{0} X$ acts on $\mathrm{Jac}_{d, L} X$. Polarizations whose multidegrees are proportional define the same compactified Jacobian, so the dependence on $L$ is only felt if $X$ is reducible.

When $X$ is irreducible but has arbitrary singularities there exists a number of deep results. For example, by [KK81] $\mathrm{Jac}_{0}$ is irreducible if and only if $X$ has planar singularities.

1.4. For us, however, the focus will be on the case when $X$ has very simple singularities - at worst nodes - but is perhaps reducible. In this case, admissible sheaves were characterized by Seshadri [Ses82]:

\section{Lemma 1.5.}

(1) A coherent sheaf $F$ of rank 1 is admissible if and only if it has the form $F=\pi_{*} F^{\prime}$ where $F^{\prime}=\pi^{*} F /$ torsion is an invertible sheaf on a partial normalization $\nu: X^{\prime} \rightarrow X$. The sheaf $F$ is not invertible precisely at the nodes $P \in X$ at which $\pi$ is not an isomorphism, and in this case the stalk $F_{P} \simeq m_{P}$, the maximal ideal of $P$.

(2) Similarly, a coherent sheaf $F$ which has rank 0 or 1 at every generic point of $X$ has the form $\pi_{*} F^{\prime}$ where $\pi: Y^{\prime} \rightarrow Y \rightarrow X$ is a partial normalization of a subcurve $Y \subset X$. The curves $Y, Y^{\prime}$ and the invertible sheaf $F^{\prime}$ are all uniquely defined by $F$.

Example 1.6. Suppose $\pi$ is a partial resolution at a single node $P$ and $\pi^{-1}(P)=\left\{P^{+}, P^{-}\right\}$. Then a simple computation shows that

$$
\pi_{*}\left(m_{P^{+}} \otimes m_{P^{-}}\right)=m_{P} \quad \text { and } \quad \pi^{*} m_{P}=\left(m_{P^{+}} \otimes m_{P^{-}}\right) \oplus k_{P^{+}} \oplus k_{P^{-}},
$$

where $k_{P^{+}}$and $k_{P^{-}}$are skyscraper sheaves.

From now on, every admissible sheaf will be assumed to have rank 0 or 1 at every generic point. By the above Lemma, to every such sheaf one can associate a unique partial normalization $Y^{\prime}$ of a subcurve $Y \subset X$ and a set of integers $\left(d_{i}\right)$, the multidegree of $F^{\prime}$ on $Y^{\prime}$. If $\operatorname{Supp} F=X$ then

$$
\sum d_{i}=\operatorname{deg} F^{\prime}=\operatorname{deg} F-\#\{P \mid \pi \text { is not an isomorphism }\}, \quad \chi(F)=\chi\left(F^{\prime}\right)
$$

(Indeed, in the example above, $\operatorname{deg} m_{P}=-1$ and $\operatorname{deg}\left(m_{P^{+}} \otimes m_{P^{-}}\right)=-2$; and $\chi\left(\mathcal{O}_{X}\right)=\chi\left(\mathcal{O}_{X^{\prime}}\right)-1$.) 
For each subcurve $Y \subset X$, let $F_{Y}$ be the maximal subsheaf of $F$ which has support $Y$. For example, if $P$ is a node at which two irreducible components $X_{1}$ and $X_{2}$ meet and $X_{1} \subset Y, X_{2} \not \subset Y$ then $F_{P} \simeq \mathcal{O}_{P}$ or $F_{P} \simeq m_{P}$ implies that $\left(F_{Y}\right)_{P} \simeq\left(i_{1}\right)_{*} m_{P_{1}}$.

If $E_{1} \subset E$ is a subsheaf such that $\operatorname{Supp} E / E_{1}$ is finite and nonempty then $\mu\left(E_{1}\right)<\mu(E)$. As a consequence, to check the (semi)stability of an admissible sheaf $F$, one has to check the slope inequality only for the finitely many subsheaves $F_{Y}$.

If $F$ is an invertible sheaf then $\operatorname{deg} F_{Y}=\operatorname{deg}\left(\left.F\right|_{Y}\right)-Y(X-Y)$, where $Y(X-Y)$ stands for the number of points in $Y \cap(X-Y)$. Hence, $F$ is semistable (resp. stable) if and only if for every proper nonempty subcurve $Y$ of $X$ one has the following inequality (resp. with $<$ ):

$$
\mu\left(F_{Y}\right)=\frac{\operatorname{deg}\left(\left.F\right|_{Y}\right)-Y(X-Y)+\chi\left(O_{Y}\right)}{\operatorname{deg}\left(\left.L\right|_{Y}\right)} \leq \frac{\operatorname{deg} F+\chi\left(O_{X}\right)}{\operatorname{deg} L}=\mu(F)
$$

If we set for simplicity $\lambda_{i}=\operatorname{deg}\left(\left.L\right|_{X_{i}}\right) / \operatorname{deg} L$ and $\lambda_{Y}=\sum_{X_{i} \subset Y} \lambda_{i}$ (so that $\left.\lambda_{X}=\sum \lambda_{i}=1\right)$ and use the fact that the degree of the dualizing sheaf $\omega_{X}$ on $Y$ is $Y(X-Y)-2 \chi\left(\mathcal{O}_{Y}\right)$ then the inequality becomes

$$
\left|\operatorname{deg}\left(\left.F\right|_{Y}\right)-\frac{1}{2} \operatorname{deg}\left(\left.\omega_{X}\right|_{Y}\right)-\lambda_{Y}\left(\operatorname{deg} F-\frac{1}{2} \operatorname{deg} \omega_{X}\right)\right| \leq \frac{1}{2} Y(X-Y)
$$

(the other half of the inequality, giving the absolute value, comes from applying the first half to the curve $X-Y$ ).

If we choose some integers $\tilde{d}_{i}$ with $\sum \tilde{d}_{i}=\operatorname{deg} F$ and define fractional numbers $\phi_{i}$ by the condition

$$
\frac{1}{2} \operatorname{deg}\left(\omega_{X} \mid X_{i}\right)+\lambda_{i}\left(\operatorname{deg} F-\frac{1}{2} \operatorname{deg} \omega_{X}\right)=\tilde{d}_{i}+\phi_{i}
$$

then the semistability condition on the multidegrees $\left(d_{i}\right)$ of $F$ becomes

$$
\sum_{X_{i} \subset Y}\left(d_{i}-\tilde{d}_{i}\right) \leq \sum_{X_{i} \subset Y} \phi_{i}+\frac{1}{2} Y(X-Y) .
$$

Hence, up to a shift, the (integral) solutions for the (semi)stable multidegrees $\left(d_{i}\right)$ depend only on the fractional parts of $\left(\phi_{i}\right)$. Note that by the definition, one has $\sum \phi_{i}=0$ since $\phi$ measures the deviation from the average.

A rank-1 sheaf $F$ that is not invertible corresponds to an invertible sheaf on a partial normalization $X^{\prime}$ of $X$, and the (semi)stability condition is the same as above but for a subcurve $Y$ of $X^{\prime}$. 
1.7. One notable special case is when the curve $X$ is stable and the polarization is canonical, i.e. $L=\omega_{X}$. Then the semistability condition (2) simplifies to

$$
\left|\operatorname{deg}\left(\left.F\right|_{Y}\right)-\lambda_{Y} \operatorname{deg} F\right| \leq \frac{1}{2} Y(X-Y) .
$$

One notes that in this case $(2 g-2) \lambda_{Y}$ are integers and $F \mapsto F \otimes \omega_{X}^{k}$ defines an isomorphism $\operatorname{Jac}_{d, \omega_{X}} X \stackrel{\sim}{\rightarrow} \operatorname{Jac}_{d+k(2 g-2), \omega_{X}} X$ for any $k \in \mathbb{Z}$.

1.8. Before plunging into combinatorics of graphs and cell decompositions, we would like to explain an alternative approach using Gieseker-semistable curves and used notably by Cornalba [Cor89, Cor91] and Caporaso [Cap94].

Let $\hat{X}$ be a curve obtained by inserting for some nodes $P \in X$ lines $E_{P} \simeq$ $\mathbb{P}^{1}$ intersecting the rest of the curve at two points; let $\hat{\pi}: \hat{X} \rightarrow X$ be the morphism contracting $E_{P}$ 's. Invertible sheaves $F$ on $X$ are in a bijection with invertible sheaves $\hat{F}$ on $\hat{X}$ such that $\operatorname{deg}\left(\left.F\right|_{E_{P}}\right)=0$. However, if $\operatorname{deg}\left(\left.F\right|_{E_{P}}\right)=1$ then $\hat{\pi}_{*} \hat{F} \simeq m_{P}$. So this is another way to encode the non-invertible sheaves. In some ways, it is more convenient because $\operatorname{deg} \hat{F}=\operatorname{deg} F$ and $\chi\left(\mathcal{O}_{\hat{X}}\right)=\chi\left(\mathcal{O}_{X}\right)$, so that the curves $C$ and $\hat{C}$ can now be fibers of the same flat family.

The price for this convenience is non-uniqueness of $\hat{F}$ (which is ruled out by the fact that $\operatorname{Pic}^{0} \hat{X}=\operatorname{Pic}^{0} X$ ). The precise statement is the following:

Lemma 1.9. Every admissible sheaf on $X$ corresponds to a unique curve $\hat{X}$ as above and a family of invertible sheaves on $\hat{X}$ with $\operatorname{deg}\left(\left.\hat{F}\right|_{E_{P}}\right)=1$ for each line $E_{P}$ forming an $\left(\mathbb{G}_{m}^{K}\right)$-orbit. Here, $K=\# P$; the multiplicative group $\mathbb{G}_{m}^{K}$ acts by scalar multiplication on each line $E_{P}$ with two marked points, 0 and $\infty$; and the orbit $\left(\mathbb{G}_{m}^{K}\right) . \hat{F}$ is for the induced action of $\mathbb{G}_{m}^{K}$ on Pic $\hat{X}$.

The sheaf $\hat{F}$ is unique if and only if every node $P$ is disconnecting, i.e. removing it increases the number of connected components of $X$.

In this approach, the (semi)stability of $F$ is equivalent to the inequality (2) applied to the sheaf $\hat{F}$ on $\hat{X}$. The inequality (5) becomes Gieseker's Basic Inequality. As a corollary, the fibers of the universal compactified jacobian $P_{d} \rightarrow \overline{\mathrm{M}}_{g}$ of [Cap94] are $\mathrm{Jac}_{d, \omega_{X}} /$ Aut $X$.

\section{§2. Combinatorics of Compactified Jacobians}

2.1. Oda and Seshadri [OS79] constructed a number of compactified jacobians $\mathrm{Jac}_{\phi}$ of nodal curves. These jacobians are exactly the same as the 
projective schemes $\mathrm{Jac}_{d, L}$ of the previous section. This may not be entirely obvious for three reasons:

(1) The semistability condition in [OS79] is formulated not in terms of subsheaves but as $\phi$-semistability, depending on a parameter $\phi \in \partial C_{1}(\Gamma, \mathbb{R})$, where $\Gamma$ is the dual graph of the curve $X$.

(2) The GIT construction differs slightly from the GIT construction used elsewhere.

(3) The result is formally stated for degree 0 only.

So, let us check that the definitions are equivalent and that the moduli spaces $\mathrm{Jac}_{d, L}$ and $\mathrm{Jac}_{\phi}$ are indeed the same. The usual scheme for constructing $\mathrm{Jac}_{d, L}$, for example employed in [Sim94], goes as follows:

(1) One fixes a Hilbert polynomial $p(n)$ and establishes that semistable admissible sheaves with $\chi(F(n))=p(n)$ form a bounded family.

(2) The semistability of $F(n)=F \otimes L^{n}$ with respect to the polarization $L$ is equivalent to semistability of $F$. By boundedness, there exists $n_{0}$ such that for $n \geq n_{0}$ the semistable sheaves $F(n)$ are globally generated and have vanishing higher cohomology.

(3) One fixes some $n \geq n_{0}$, a vector space (or a free $\mathcal{O}_{S}$-module) $E$ of rank $p(n)$ and considers the Grothendieck's Hilbert scheme $\operatorname{Quot}\left(E \otimes \mathcal{O}_{X}, p\right)$ parameterizing admissible quotients of $E \otimes \mathcal{O}_{X}$ which have the given polynomial $p$.

(4) At this step, there is a choice. One usually embeds Quot into a Grassmanian which in turn embeds into a projective space by Plücker coordinates. Then one looks at the Hilbert-Mumford criterion for (semi)stability for the PGLaction on projective spaces and shows that for $n \gg 0$ it is equivalent to the (semi)stability of sheaves of the previous Section, in terms of subsheaves.

(5) Hence, semistable (resp. stable) sheaves form PGL-equivariant open subsets, and by taking the GIT quotients one obtains a projective coarse moduli space (resp. a quasi-projective fine moduli space) of semistable (resp. stable) sheaves.

The approach of [OS79] differs from this only in Step 4. Instead of embedding Quot into a Grassmanian, they choose a large number of points $x_{1}, \ldots$, $x_{N} \in X$ and embed Quot into a product of $N$ projective spaces $\prod \mathbb{P}\left(E^{*}\right)$ by 
looking at 1-dimensional quotients $E \stackrel{\sim}{\rightarrow} H^{0}(F(n)) \rightarrow H^{0}\left(\left.F(n)\right|_{x_{i}}\right)$. Then they use the Hilbert-Mumford criterion for the PGL $(E)$-action on this product.

The relation with the other setup is as follows, where we refer the reader to the Outline of the main argument on pp.6-8 of [OS79]. Oda and Seshadri show that a sheaf $F$ is (semi)stable in their setup iff for every proper subcurve $Y \subset X$ one has inequality $(*)$, equivalently $(* *)$ on p.7.

Now, choose the numbers of points $\left(N_{i}\right), \sum N_{i}=N$, on irreducible components of $X$ and also the auxiliary parameters $\left(\tilde{n}_{i}\right)$ to be proportional to the multidegree of the polarization, i.e. to $\left(\lambda_{i}\right)$. Then the inequality $(*)$ is just our inequality (1) for the subsheaves $F_{Y}$ and (**) is the inequality (2). The definition of the parameter $\phi \in \partial C^{1}(\Gamma, \mathbb{Q})$ in [OS79] coincides with our formula (3). Hence, $\mathrm{Jac}_{d, L} \simeq \mathrm{Jac}_{\phi}$, where $\phi=\phi\left(d, \lambda_{i}\right)$ is defined by the formula (3). So we have a map $(d, L) \rightarrow \phi \in \partial C_{1}(\mathbb{Q})$ and it is also easy to see that this map is surjective.

One further remark is that [OS79] concentrates on the case of $\operatorname{deg} F=$ 0 . But as this outline already shows, the moduli spaces in [OS79, §11] are constructed for semistable sheaves of every degree.

2.2. Let us now turn to the combinatorics of the compactified jacobians. We will use the following customary notaion:

(1) If $X$ is a nodal curve then $\Gamma=\Gamma(X)$ is the dual graph with vertices $\left\{v_{i} ; i \in I\right\}$ corresponding to irreducible components $X_{i}$ of $X$ and edges $\left\{e_{j} ; j \in J\right\}$ corresponding to nodes. We fix an orientation of $\Gamma$.

(2) $\pi: \widetilde{X} \rightarrow X$ denotes the normalization of $X, \widetilde{X}_{i}$ are normalizations of $X_{i}$.

(3) $g=p_{a}(X)$ is the arithmetic genus of $X, g_{i}=p_{a}\left(X_{i}\right), \tilde{g}_{i}=p_{a}\left(\tilde{X}_{i}\right)$.

(4) $C_{0}, C_{1}, C^{0}, C^{1}, H_{0}, H_{1}, H^{0}, H^{1}$ denote the spaces of chains, cochains and (co)homologies of the graph $\Gamma$, with integral coefficients.

$$
\partial: C_{1} \rightarrow C_{0}, \quad \text { edge } e \mapsto \operatorname{end}(e)-\operatorname{beg}(e)
$$

is the boundary operator defined by the chosen orientation.

(5) $h_{1}(X)=\operatorname{dim} H_{1}(\Gamma)$ is the cyclotomic number of the graph $\Gamma(X)$.

The ordinary Jacobian is $\mathbf{P i c}{ }^{0} X$, the fine moduli space of invertible sheaves of multidegree $(0, \ldots, 0)$. This is a group variety acting on all compactified jacobians, and the following result is basic (for the proof, see f.e. [OS79, 10.2]). 
Theorem 2.3. $\quad \mathbf{P i c}^{0} X$ is a semiabelian variety, an extension of an abelian variety $\mathbf{P i c}^{0} \tilde{X}$ by a multiplicative torus whose character group is $H_{1}(\Gamma, \mathbb{Z})$. For the geometric points, we have:

$$
1 \rightarrow H^{1}\left(\Gamma, k^{*}\right) \rightarrow \operatorname{Pic}^{0} X \stackrel{\pi^{*}}{\longrightarrow} \operatorname{Pic}^{0} \tilde{X} \rightarrow 0
$$

(Let $P \in X$ be a node. Consider an invertible regular function $f$ defined on a neighborhood $U \subset \widetilde{X}$ of $\pi^{-1}(P)=\left\{P^{+}, P^{-}\right\}$such that $f\left(P^{+}\right) / f\left(P^{-}\right)=z \in$ $k^{*}$. Then $f$ defines a local section of $\mathcal{K}_{X}^{*} / \mathcal{O}_{X}^{*}$, i.e. a Cartier divisor supported at $P$ and usually denoted $(z, 0,0)_{P}$. Such Cartier divisors are parameterized by $C^{1}\left(\Gamma, k^{*}\right)$. The group $C^{0}\left(\Gamma, k^{*}\right)$ parameterizes principal Cartier divisors given by locally constant functions $f$ on $\widetilde{X}$. The homomorphism $C^{1} \rightarrow \operatorname{Pic}^{0} X$ induces the embedding $H^{1} \rightarrow \mathrm{Pic}^{0} X$.

2.4. An extension of an abelian variety $A$ by a torus $T \simeq \mathbb{G}_{m}^{r}$ is equivalent to a homomorphism $c^{t}: \Lambda(T) \simeq \mathbb{Z}^{r} \rightarrow A^{t}=\mathrm{Pic}^{0} A$ from the character group of $T$ to the dual abelian variety of $A$. In our case, the dual abelian variety is identified with $\mathbf{P i c}^{0} \widetilde{X}$ via the principal polarization on the Jacobian of $\widetilde{X}$, and the character group of the torus is $H_{1}(\Gamma, \mathbb{Z})$. The homomorphism is described explicitly as follows.

Every edge $e$ of the graph $\Gamma$ corresponds to a node $P$ of $X$, and the orientation defines an ordered pair of points $\left(P^{-}, P^{+}\right)$on $\widetilde{X}$. Let $c^{t}(e)=$ $P^{+}-P^{-} \in \operatorname{Pic} \tilde{X}$, and extend this by linearity to $C_{1}(\Gamma, \mathbb{Z})$. Then if $h \in H_{1}$ then $c^{t}(h) \in \operatorname{Pic}^{0} \widetilde{X}$ and that is the required homomorphism.

2.5. In the space $C_{1}(\Gamma, \mathbb{R})=\oplus_{j \in J} \mathbb{R} e_{j}$ with the lattice $C_{1}(\Gamma, \mathbb{Z})$ one defines two infinite periodic decompositions:

(1) The Delaunay decomposition Del consisting of standard cubes cut out by hyperplanes $z_{j}=n_{j}$ for $n_{j} \in \mathbb{Z}$.

(2) The Voronoi decomposition Vor which in this case is the Delaunay decomposition shifted by the vector $e(J) / 2=\sum_{j \in J} e_{j} / 2$.

These two decompositions are in a dual relationship: for every Delaunay cell, a cube $D$ of dimension $0 \leq \operatorname{dim} D \leq|J|$, the dual Voronoi cell is the unique cube $V=D^{*}$ of the complementary dimension such that $D \cap V$ is a point.

(For the background: For every vector space with a positive definite quadratic form and a lattice one defines Delaunay and Voronoi decompositions which are dual in the same sense as above. Vertices of polytopes of the Delaunay decomposition are always in the lattice, and for the Voronoi decomposition 
they are not. If one varies the form $q$, $\operatorname{Vor}(q)$ changes continuously but the set of possibilities for $\operatorname{Del}(q)$ is discrete.)

2.6. For every $\phi \in \partial C_{1}(\mathbb{Q}) \subset C_{0}(\mathbb{Q})$, let $H_{1}(\mathbb{R})_{\phi}$ denote the preimage $\partial^{-1}(\phi)$, an affine subspace of $C_{1}(\mathbb{R})$ which is parallel to $H_{1}(\mathbb{R})$. To this, one can associate two further polyhedral decompositions:

(1) The decomposition $\operatorname{Vor}_{\phi}=\operatorname{Vor} \cap H_{1}(\mathbb{R})_{\phi}$ of $H_{1}(\mathbb{R})_{\phi}$ into rational polytopes, periodic with only finitely many polytopes modulo $H_{1}(\mathbb{Z})$.

(2) The decomposition $\operatorname{Del}_{\phi}$ called in [OS79] the Namikawa decomposition defined as follows: for each Voroni cell $V \in$ Vor with $\partial(V) \ni \phi$, consider the dual Delaunay cell $V^{*}$ and its orthogonal projection $\partial^{\perp}\left(V^{*}\right)$ to $H_{1}$ with respect to the standard Euclidean form on $C_{1}$. The images form a polyhedral decomposition with vertices in $\partial^{\perp}\left(C_{1}(\mathbb{Z})\right.$ and periodic with only finitely many polytopes modulo $H_{1}(\mathbb{Z})$.

By $[$ OS79, 1.5] these two decompositions also stand in duality. Denote by $\overline{\operatorname{Vor}}_{\phi}=\operatorname{Vor}_{\phi} / H_{1}(\mathbb{Z}), \overline{\operatorname{Del}}_{\phi}=\operatorname{Del}_{\phi} / H_{1}(\mathbb{Z})$ the quotient complexes.

In toric geometry it is customary to denote the character ("monomial") lattice of the torus $T$ by $M$ and the lattice of 1-parameter subgroups of $T$ by $N$. Fans live in the $N$-space and polytopes for projective toric varieties live in the $M$-space.

For what follows, it is important to think of $\mathrm{Del}_{\phi}$ as living in the $N$-space and being a generalized version of a fan, and of $\operatorname{Vor}_{\phi}$ as living in the $M$-space and being an honest collection of rational polytopes defining projective toric varieties.

2.7. Let us emphasize the following: the decomposition $\operatorname{Vor}_{\phi}$ is a $H_{1}(\mathbb{Z})$ periodic decomposition of $H_{1}(\mathbb{R})$ by hyperplanes corresponding to the edges $e_{j}$ of the graph $\Gamma$ which lie on least one cycle. Two edges $e_{j}, e_{j^{\prime}}$ define the same hyperplane if they are equivalent with respect to $H_{1}$, i.e. $e_{j}$ lies on a cycle iff so does $e_{j^{\prime}}$.

Remark 2.8. It is unfortunate that in the important special case $\phi=$ $\partial e(J) / 2$ the decomposition $\operatorname{Vor}_{\phi}$ is the Delaunay decomposition for the lattice $e(J) / 2+H_{1}(\mathbb{Z})$ and the quadratic form $q$ which is the restriction of the standard Euclidean form from $C_{1}(\mathbb{Z})$; and $\operatorname{Del}_{\phi}$ is the corresponding Voronoi decomposition.

The notations $\mathrm{Del}_{\phi}, \mathrm{Vor}_{\phi}$ in [OS79] are motivated by a general construction which applies to lattices not necessarily coming from a graph. In the case of $C_{1}$ 
with the standard Euclidean quadratic form, the difference between Vor and Del is only a shift by $\partial e(J) / 2$ but it seems that it would be better to switch them. Life sometimes gets confusing when there are two many canonical selfdualities!

To each polytope $\delta$ in the decomposition $\operatorname{Vor}_{\phi}$ one can associate a projective toric variety $P_{\delta}$ and an ample $\mathbb{Q}$-line bundle. The torus $T$ acting on this toric variety is $H^{1}\left(\Gamma, k^{*}\right)$, the toric part of $\operatorname{Pic}^{0} X$.

If $\delta$ is maximal-dimensional then the dual cell $\delta^{*}$ in the Namikawa decomposition is a point. If one looks at a small neighborhood of this point, one obtains a complete fan. This is precisely the normal fan of the toric variety $P_{\delta}$.

Let $Z_{\delta}$ be the twisted fiber product $P_{\delta} \times_{T} \operatorname{Pic}^{0} X=\left(P_{\delta} \times \operatorname{Pic}^{0} X\right) / T$, with $t \in T$ acting on $P_{\delta}$ in the standard way and on $P i c^{0}$ by multiplying by $t^{-1}$. The following is the main structure theorem of [OS79] about $\mathrm{Jac}_{\phi}$ :

\section{Theorem 2.9.}

(1) $\mathrm{Jac}_{\phi} X$ is reduced projective scheme.

(2) For each $\delta \in \overline{\operatorname{Vor}}_{\phi}$ there exists a finite morphism $Z_{\delta} \rightarrow \mathrm{Jac}_{\phi}$. The images cover $\mathrm{Jac}_{\phi}$ and the inclusion relations between them are the same as in $\overline{\operatorname{Vor}}_{\phi}$. In particular, $\mathrm{Jac}_{\phi}$ is connected and its normalization coincides with the union of $Z_{\delta}$ for the maximal-dimensional polytopes $\delta$.

Proof. (1) [OS79, Thm.11.4] is and (2) is [OS79, Thm.13.2].

Remark 2.10. As $\phi$ varies, the varieties $\mathrm{Jac}_{\phi}$ change according to the changing configuration of polytopes of the slice $\operatorname{Vor} \cap H_{1}(\mathbb{R})_{\phi}$. The situation is entirely similar to the way a GIT quotient of a toric variety is changing when one varies the polarization, see f.e. [DH98, KSZ91]. For a non-rational $\phi$ the decomposition Vor $_{\phi}$ defines a symplectic form on the nonsingular part of $\mathrm{Jac}_{\phi}$ (provided one works over $\mathbb{C}$ ) but not a polarization.

This is another case where a phenomenon appeared first in the "infinite periodic" case before being picked up in the "finite" toric case. Another example is the implicit appearance of the secondary fan in the work of Voronoi [Vor09].

\section{§3. Canonical Compactified Jacobian $\mathrm{Jac}_{g-1}$}

Let us start with the following very simple observation:

Lemma 3.1. $\quad \operatorname{Jac}_{g-1, L} X$ does not depend on the polarization $L$. 
Proof. Indeed, the condition $\operatorname{deg} F=g-1$ is equivalent to $\chi(F)=0$. Then the slope $\mu(F)=\chi(F) / \sum l_{i}$ is zero and the condition $\mu(E) \leq \mu(F)$ is equivalent to the condition $\chi(E) \leq 0$. In other words, it does not depend on the multidegree of $L$.

Alternatively, the inequality (2) becomes

$$
\left|\operatorname{deg}\left(\left.F\right|_{Y}\right)-\frac{1}{2} \operatorname{deg}\left(\left.\omega_{X}\right|_{Y}\right)\right| \leq \frac{1}{2} Y(X-Y),
$$

i.e. it no longer involves $\lambda_{Y}$.

For this reason we will call $\mathrm{Jac}_{g-1} X$ the canonical compactified jacobian, although it does not contain $\mathbf{P i c}^{0} X$ unless $g=1$.

$\mathrm{Jac}_{g-1}$ admits a very nice description in terms of the orientations on the graph $\Gamma$, as follows.

Definition 3.2. $\quad$ A subgraph $\Gamma^{\prime} \subset \Gamma$ is said to be generating if vertices $(\Gamma)=\operatorname{vertices}\left(\Gamma^{\prime}\right)$. Every such subgraph corresponds to a partial normalization of $X$ at the nodes $\Gamma-\Gamma^{\prime}$. We denote this partial normalization by $\pi\left(\Gamma^{\prime}\right)$ : $X\left(\Gamma^{\prime}\right) \rightarrow X$. Note in particular that $X(\Gamma)=X$ and that $\tilde{X}$ is $X\left(\Gamma^{\prime}\right)$ where $\Gamma^{\prime}$ has all the vertices of $\Gamma$ but no edges at all.

Definition 3.3. $\quad$ A subgraph $\Gamma^{\prime} \subset \Gamma$ is said to be complete if vertices $\left(\Gamma^{\prime}\right)$ $\subset$ vertices $(\Gamma)$ and edges $\left(\Gamma^{\prime}\right)$ are precisely the edges of $\Gamma$ lying inside $\Gamma^{\prime}$. These graphs correspond to subcurves $Y \subset X$. We may identify such subcurves $Y$ with the corresponding subgraphs.

Definition 3.4. If $\underline{d}=\left(d_{i}\right)$ is a multidegree of total degree $\sum d_{i}=g-1$ then its normalized multidegree $\underline{e}$ is defined by $e_{i}=d_{i}-\left(\tilde{g}_{i}-1\right)$. Note that $\sum e_{i}$ equals the number of edges of $\Gamma$.

For a subcurve $Y \subset X$, i.e. a complete subgraph $\Gamma^{\prime} \subset \Gamma$, we set

$$
d_{Y}=\sum_{X_{i} \subset Y} d_{i}, \quad e_{Y}=\sum_{X_{i} \subset Y} e_{i}
$$

Definition 3.5. We will call a multidegree $\underline{d}$ (and the normalized multidegree $\underline{e}$ ) semistable (resp. stable) if the sheaves of this degree are semistable (resp. stable).

Proposition 3.6. A normalized multidegree $\underline{e}$ is semistable (resp. stable) if any of the following equivalent conditions hold: 
(i) For every subcurve $Y \subset X$, one has

$$
\left|e_{Y}-\# \operatorname{edges}(Y)-\frac{1}{2} Y(X-Y)\right| \leq \frac{1}{2} Y(X-Y), \quad \text { resp. } \quad<
$$

(ii) $e_{Y} \leq \#$ edges $(Y)+Y(X-Y)$, resp. $<$.

(iii) There exists an orientation of the graph $\Gamma$ such that $e_{i}$ equals the number of arrows pointing at $X_{i}$ (resp. in addition $X$ is connected and there is no proper subcurve $Y \subset X$ such that all arrows between $Y$ and $X-Y$ go in one direction).

Proof. Condition (i) is just the restatement of our basic inequality. The implication (i) $\Rightarrow$ (ii) is clear and the inverse is obtained by looking at $Y^{\prime}=$ $X-Y$. (iii) obviously implies (ii).

To prove the implication (ii) $\Rightarrow$ (iii) first assume that the normalized multidegree $\underline{e}$ of the graph of $X$ is strictly semistable, i.e. there exists a subcurve $Y \subset X$ for which the equality holds. Then consider separately the following multidegrees on $Y$ and $X-Y$. On $X-Y$ simply take the restriction of $\underline{e}$. On $Y$, however, for every vertex $X_{i}$ take $e_{i}^{\prime}=e_{i}$ minus the number of edges between $X_{i}$ and $X-Y$. Then it is easy to show that the two multidegrees thus obtained are semistable. Therefore, the orientations on $Y$ and $X-Y$ exist by the induction on the number of vertices. To complete the orientation of $X$, orient all the edges between $Y$ and $X-Y$ to point at $Y$.

In general, starting with a semistable multidegree as in (ii) we can fix an arbitrary vertex $X_{i_{0}}$ and change the degrees of $X_{i_{0}}$ and the neighboring vertices = curves $X_{j}$ by 1 to make the multidegree strictly semistable, thus reducing to the previous case. Hence, we get an orientation for the modified multidegree. The orientation for the original multidegree is then obtained by reversing the orientations of edges $\left(i_{0}, j\right)$.

The third condition of the above definition is the easiest to check. We will call an orientation satisfying (iii) semistable (resp. stable). Note that different orientations may well produce the same multidegree.

Let us now summarize our discussion as follows:

\section{Theorem 3.7.}

(1) $F$ is semistable iff the normalized multidegree $\left(\underline{e}^{\prime}\right)$ on the corresponding to $F$ spanning subgraph $\Gamma^{\prime}$ is semistable.

(2) Assume that the curve $X$ is connected. Then $F$ is stable iff $\left(\underline{e}^{\prime}\right)$ is stable. 
(3) $\mathrm{Jac}_{g-1} X$ has a natural stratification into homogeneous spaces over $\mathrm{Pic}^{0} X$. Each stratum corresponds in a 1-to-1 way to a stable multidegree $\underline{d^{\prime}}$ (resp. stable normalized multidegree $\left.\underline{e}^{\prime}\right)$ on a generating subgraph $\Gamma^{\prime} \subset \Gamma$. The $k$-points of this stratum can be identified with $k$-points of $\operatorname{Pic}_{\underline{d}^{\prime}}\left(X\left(\Gamma^{\prime}\right)\right)$, i.e. with invertible sheaves on $X\left(\Gamma^{\prime}\right)$ of multidegree $\underline{d^{\prime}}$. The codimension of this stratum equals $h_{1}(\Gamma)-h_{1}\left(\Gamma^{\prime}\right)$.

The following statement is the first step towards constructing the theta divisor on $\mathrm{Jac}_{g-1}$, which will be completed in Theorem 5.3.

Lemma 3.8. For a multidegree $\underline{d}=\left(d_{i}\right)$ with $\sum d_{i}=g-1$, the subset

$$
\left\{s \in \operatorname{Pic}^{\underline{d}} X \mid h^{0}\left(F_{s}\right)>0\right\} \subset \operatorname{Pic}^{\underline{d}} X
$$

is a divisor if and only if $\underline{d}$ is semistable.

Proof. This follows by [Bea77, 2.1] and the part (iii) of Definition-Proposition 3.6.

Remark 3.9. This gives a yet another characterization of semistable admissible sheaves in degree $g-1$ : they have the multidegrees for which the usual definition of the theta-divisor actually gives a divisor.

3.10. The decomposition $\operatorname{Vor}_{\phi}$ in the case of degree $g-1$ is very special. The formula $(3)$ says that $\phi_{i}=(1 / 2) \operatorname{deg}\left(\left.\omega_{X}\right|_{X_{i}}\right)(\bmod \mathbb{Z})$. This means that $\phi_{i}$ is $1 / 2(\bmod \mathbb{Z})$ if the degree of the vertex $v_{i}$ is odd and $0(\bmod \mathbb{Z})$ if it is even. In other words, the degree $g-1$ case corresponds to the choice $\phi=\partial e(J) / 2$.

After shifting the cubes in the Voronoi decomposition of $C_{1}(\mathbb{R})$ by $-\partial e(J) / 2$ to the origin, we see that $\operatorname{Vor}_{\phi}$ is obtained by intersecting the subspace $H_{1}(\mathbb{R}) \subset C_{1}(\mathbb{R})$ by systems of parallel hyperplanes $z_{j}=n_{j} \in \mathbb{Z}$, where $z_{j}, j=1, \ldots,|J|$ are the coordinate functions on $C_{1}$. Let $e_{1}^{*}, \ldots, e_{|J|}^{*} \in H_{1}^{*}$ be the vectors in the dual lattice defining the linear functions $\left.z_{j}\right|_{H_{1}}$. The following is a basic fact from graph theory:

Theorem 3.11. The system of vectors $\left\{e_{1}^{*}, \ldots, e_{|J|}^{*}\right\} \subset H_{1}^{*}$ is totally unimodular, i.e. any subset which is a basis of $C_{1}^{*}(\mathbb{R})$ is also a basis of the lattice $C_{1}^{*}$. Equivalently, the 0 -skeleton of the hyperplane arrangement $\left\{e_{j}^{*}=n_{j} \in \mathbb{Z}\right\}$ is the lattice $H_{1}$ itself.

In matroid theory, the totally unimodular system of this form are called cographic regular matroids, see f.e. [Oxl92]. 
3.12. As already noted in [OS79], the compactified jacobian $\operatorname{Jac}_{\partial e(J) / 2}$ coincides with the stable quasiabelian variety which Namikawa associated to a nodal curve in [Nam76].

\section{§4. Extended (Toroidal) Torelli Map}

The following theorem is due to Mumford. The proof is contained in [Nam76]; it is purely combinatorial and does not use moduli interpretations of the spaces involved. We outline the argument below.

Theorem 4.1. The classical Torelli map $\mathrm{t}: \mathrm{M}_{g} \rightarrow \mathrm{A}_{g}$ can be extended to a regular map from the Deligne-Mumford compactification $\overline{\mathrm{M}}_{g}$ to the toroidal compactification $\overline{\mathrm{A}}_{g}^{\mathrm{Vor}}$ for the 2nd Voronoi fan.

Sketch of the proof. Let $\overline{\mathrm{A}}_{g}^{\tau}$ be the toroidal compactification of $\mathrm{A}_{g}$ corresponding to any admissible $\operatorname{GL}(g, \mathbb{Z})$-invariant fan $\tau$ whose support equals the convex hull of rational positive semidefinite quadratic forms on $\mathbb{Z}^{g}$. The stacks $\overline{\mathcal{M}}_{g}, \overline{\mathcal{A}}_{g}^{\tau}$ are toroidal, and the coarse moduli spaces $\overline{\mathrm{M}}_{g}, \overline{\mathrm{A}}_{g}^{\tau}$ are locally quotients of toroidal varieties by finite groups. Hence, the rational map $\overline{\mathfrak{t}}: \overline{\mathrm{M}}_{g} \rightarrow \overline{\mathrm{A}}_{g}^{\tau}$ is regular if and only if every cone in the first fan maps inside of a cone in the second fan.

For a neighbourhood of a stable curve $[C] \in \overline{\mathrm{M}}_{g}$, the cone in the first fan can be identified with quadrant $\oplus_{i=1}^{n} \mathbb{R}_{\geq 0} e_{j}$, where $e_{j}$ 's correspond to the nodes of $C$. The second cone lies in the cone of positive semidefinite quadratic form on $H_{1}(\Gamma, \mathbb{R})$. As in the previous section, let $z_{j}$ be restrictions to $H_{1}$ of the coordinate functions on $C_{1}$. Then the map of fans sends $e_{j}$ to $z_{j}^{2}$. Thus, the rational map $\overline{\mathfrak{t}}$ is regular if and only if all $z_{j}^{2}$ lie in a common cone of the fan $\tau$.

The second Voronoi fan $\tau^{\text {Vor }}$ has this property. Indeed, by definition, a locally closed cone of $\tau^{\text {Vor }}$ consists of quadratic forms $q$ on $H_{1}(\Gamma)$ defining the same Delaunay decomposition. For any primitive integral linear functions $f_{1}, \ldots$ the Delaunay decomposition of $\sum c_{k} f_{k}^{2}$ does not depend on the choice of positive $c_{k}$ if and only if the corresponding system of vectors in the dual space is totally unimodular. For the functions $z_{1}, \ldots z_{|J|}$ this is just Theorem 3.11.

\section{$\S 5 . \quad$ Compactified Jacobians as Stable Varieties and Pairs}

In [Ale02], we defined a stable semiabelic variety as a connected reduced projective $G$-variety $P$ for a semiabelian group variety $G$ which satisfies the following conditions: 
(1) (on singularities) $P$ is seminormal

(2) (on group action) the number of $G$-orbits is finite, and the stabilizer of every geometric point is connected, reduced and lies in the toric part $T$ of $G$.

A stable pair $(P, D)$ has in addition an ample effective divisor $D$ satisfying the following crucial condition:

(3) $D$ does not contain any $G$-orbit entirely.

Theorem 5.1. $\quad$ Every compactified jacobian $\mathrm{Jac}_{\phi}$ is a stable semiabelic variety for the semiabelian group variety $G=\mathbf{P i c}^{0} X$.

Proof. The variety $\mathrm{Jac}_{\phi}$ is projective, connected and reduced by Theorem 2.9. There are only finitely many semistable multidegrees with a fixed $d=\sum d_{i}$. For each multidegree the semistable sheaves give a unique orbit under $\mathbf{P i c}^{0} X$ and the stabilizer is a subtorus of the toric part.

The hard part is seminormality. We know that $\mathrm{Jac}_{\phi}$ is a GIT quotient $R / /$ PGL of an open subset $R$ of a certain Hilbert scheme. We claim that $R / /$ PGL is seminormal if so is $R$. Indeed, let $Z$ be the unique seminormalization of $R / /$ PGL. Then the morphism $R \times_{R / / \mathrm{PGL}} Z \rightarrow R$ is finite and bijective, and so has to be an isomorphism if $R$ is seminormal. Hence, $Z \rightarrow R / /$ PGL is an isomorphism.

So we are reduced to showing that $R$ is seminormal. In the course of proving that $R$ is reduced Oda and Seshadri establish [OS79, p.60] the following: There exists an open subscheme $Y \subset R \times \mathbb{P}$ which projects surjectively to the first summand and a formally smooth morphism $Y \rightarrow H$ to the Hilbert scheme $H=H_{d}$ parameterizing reduced length- $d$ subschemes of the curve $X$. This obviously implies that $R$ is seminormal $\Longleftrightarrow$ so is $Y \Longleftrightarrow$ so is $H$. Finally, $H$ is a quotient of an open subset of $X \times \cdots \times X$ by the symmetric group, and so is obviously seminormal.

For any projective family $X \rightarrow S$ with a relatively ample sheaf $L$ and a chosen Hilbert polynomial $p(n)$, Theorem 1.2 gives a projective family $\mathrm{M}_{p} X \rightarrow$ $S$ of moduli spaces of semistable sheaves. In general, there is no expectation for this family to be flat. For the compactified jacobians of stable curves, however, this does hold:

Theorem 5.2. For any $d \in \mathbb{Z}$ and any flat family of stable curves $X \rightarrow$ $S$, the associated family $\mathrm{Jac}_{d, L} X \rightarrow S$ is flat. 
Proof. Even though the moduli space $\overline{\mathrm{M}}_{g}$ of stable curves is only a coarse moduli space, it is well-known that any family $X \rightarrow S$ of stable curves can étale locally be obtained by pullback from a "versal" family $X_{U} \rightarrow U$ with reduced $U$. Hence, it is sufficient to prove flatness for flat families of curves over reduced bases. Choose a relatively ample sheaf on $\operatorname{Jac}_{d, L} X \rightarrow S$. Then the flatness is equivalent to the constancy of the Hilbert polynomial. This can be checked on generic one-parameter families $s \in C \subset S$ through the point $s$, and for these the flatness follows because the fibers $\mathrm{Jac}_{d, L_{s}} X_{s}$ are reduced, connected and of the same dimension $p_{a}\left(X_{s}\right)$.

Hence, all compactified jacobians $\mathrm{Jac}_{d, L}$ are degenerations of abelian varieties. In the case $d=g-1$ they are even degenerations of principally polarized abelian varieties:

Theorem 5.3. $\quad$ For any nodal curve $X, \mathrm{Jac}_{g-1} X$ comes with a natural ample Cartier divisor $\Theta$. If $X \rightarrow S$ is a family of stable curves then $\Theta$ is a relative ample Cartier divisor.

Proof. First, we would like to see that the condition $h^{0}\left(X, F_{s}\right) \neq 0$ defines a closed subscheme of $\mathrm{Jac}_{g-1} X$ defined locally by one equation. We use an argument of Soucaris and Esteves [Sou94, Est95] which they applied to irreducible curves.

Let $F$ be a semistable sheaf on $X \times S$ with $\chi\left(F_{s}\right)=0$. By [KM76], there a natural line bundle $L(F)=\left(\operatorname{det} R \pi_{*} F\right)^{-1}$ and since $\chi\left(F_{s}\right)=0$, it comes with a canonical section $\theta$. Moreover, this works for families of curves as well.

If we replace $F$ by $F \otimes \pi^{*} E, L(F)$ will be replaced by $L(F) \otimes E^{-\chi\left(F_{t}\right)}=$ $L(F)$, so it is universally defined. Moreover, two gr-equivalent families of semistable sheaves define the same $L(F)$. The latter follows from the fact that if

$$
0 \rightarrow F^{\prime} \rightarrow F \rightarrow F^{\prime \prime} \rightarrow 0
$$

is an exact sequence, then $\operatorname{det} R \pi_{*} F=\left(\operatorname{det} R \pi_{*} F^{\prime}\right) \otimes\left(\operatorname{det} R \pi_{*} F^{\prime \prime}\right)$, so only the stable factors are important. $\mathrm{Jac}_{g-1}$ is constructed using GIT as a quotient of the Grothendieck's Quot-schemes. The above property implies that the sheaf $L(F)$ and its section descends to $\mathrm{Jac}_{g-1}$.

Since $\mathrm{Jac}_{g-1} X$ is reduced, the section $\theta$ is locally a non-zerodivisor if and only if its zero set does not contain an irreducible component. This follows by Lemma 3.8.

Let $g \in \operatorname{Pic}^{0} X$ be an $n$-torsion element. Then the divisors $n \Theta$ and $n(g . \Theta)$ are linearly equivalent. Using Lemma 3.8 again, we see that some multiple $n \Theta$ 
is basepoint-free and hence defines a morphism, call it $\varphi$, to a projective space. Because of the group action, if $\varphi$ is not injective then it has to contract an orbit of positive dimension, which is ruled out by Lemma 3.8 one again. Hence, $\varphi$ is injective, and $\Theta$ is ample.

Corollary 5.4. The functor $(X \rightarrow S) \mapsto\left(\left(\operatorname{Jac}_{g-1} X, \Theta\right) \rightarrow S\right)$ defines a morphism from the moduli space $\overline{\mathrm{M}}_{g}$ of stable curves to the moduli space $\overline{\mathrm{AP}}_{g}$ of [Ale02] of stable semiabelic pairs.

By [Ale02], the main irreducible component of $\overline{\mathrm{AP}}_{g}$ is $\overline{\mathrm{A}}_{g}^{\mathrm{Vor}}$.

5.5. Now that we know that all $\mathrm{Jac}_{\phi}, \mathrm{Jac}_{d, L}$ are stable semiabelic varieties which are degenerations of abelian varieties, we can combine the description of Theorem 2.9 with that of [Ale02]. By [Ale02], polarized stable semiabelic pairs correspond to the following combinatorial data:

(1) A lattice $\Lambda \simeq \mathbb{Z}^{r}$ and a sublattice $i: Y \rightarrow \Lambda$.

(2) A cell complex $\Delta$ with a finite reference map $\rho:|\Delta| \rightarrow \Lambda_{\mathbb{R}}$ identifying each cell $\delta$ with a lattice polytope. The complex $\Delta$ must have a $Y$-action compatible with the translation action of $Y$ on $\Lambda_{\mathbb{R}}$, and the quotient $\Delta / Y$ must be finite.

(3) The gluing data which is an element of a certain cohomology group $H^{1}$ with values in a $\mathbb{G}_{m}$-torsor.

For varieties $\mathrm{Jac}_{\phi}$, this gives the following:

(1) The lattice $\Lambda$ is $H_{1}$. Depending on the polarization chosen, the lattice $Y$ is $N H_{1}$ where $N$ is such that the polytopes of $\operatorname{Vor}_{\phi}$ become integral after multiplying by $N$. (For example, for the canonically polarized jacobian one can take $N=1$ and $Y=\Lambda=H_{1}$.)

(2) The complex of polytopes is just $N \operatorname{Vor}_{\phi}$.

(3) The gluing data in this case consists of three parts:

(a) A homomorphism $c^{t}: \Lambda \rightarrow\left(\mathbf{P i c}^{0} \widetilde{X}\right)^{t}$ defining the extension $1 \rightarrow T \rightarrow$ $\mathbf{P i c}^{0} X \rightarrow \mathbf{P i c}^{0} \widetilde{X} \rightarrow 0$, which we described it in 2.4 .

(b) A homomorphism $c: Y \rightarrow \mathbf{P i c}^{0} \tilde{X}$ satisfying $c^{t} \circ i=\lambda \circ c: Y \rightarrow$ $\left(\mathbf{P i c}{ }^{0} \tilde{X}\right)^{t}$, where $\lambda$ is the principal polarization. Hence, $c$ is just the restriction of $c^{t}$ to a sublattice. 
(c) The equivalence class of the bihomomorphism $\tau_{0}: Y \times \Lambda \rightarrow\left(c^{t} \times\right.$ c) ${ }^{*} \mathcal{P}^{-1}$ with values in the inverse of the Poincare biextension. This is given by the so called Deligne symbol, a generalization of the double ratio (see $[\mathrm{BM} 86])$.

\section{$\S 6 . \quad$ Kapranov's Compactification}

In [Kap93] Kapranov constructed a compactification of the configuration space of $n$-points (or, dually of $n$ hyperplanes) in a projective space that are in general position. We would like to relate this compactification to a "finite" toric analog of the extended toroidal Torelli map.

The map that we are interested in is explicit in Kapranov's work. We are going to modify the target of this map slightly and interpret the source and the target as moduli spaces of stable pairs.

Definition 6.1. Let $\operatorname{Gr}(r, n)$ denote the Grassmanian of $r$-dimensional linear subspaces of $\mathbb{C}^{n}$. Fix the standard coordinates $x_{1}, \ldots x_{n}$ on $\mathbb{C}^{n}$ and let $\operatorname{Gr}^{0}(r, n)$ denote the open subset parameterizing vector spaces $V$ with

$$
V \cap \cap_{\alpha \in I}\left\{x_{\alpha}=0\right\}=0
$$

for all $I \subset\{1, \ldots, n\}$ of cardinality $r+1$.

The torus $T=\left(\mathbb{C}^{*}\right)^{n} / \mathbb{C}^{*}$ acts freely on $\operatorname{Gr}^{0}(r, n)$. We will denote by $\mathrm{KS}_{r, n}$ the quotient $\operatorname{Gr}^{0}(r, n) / T$.

Via the Gelfand-McPherson correspondence, points of $\mathrm{KS}_{r, n}$ are in a bijection with isomorphism classes of pairs $\left(\mathbb{P}^{r-1}, E\right)$, where $E=\sum H_{i}$ is a union of $n$ hyperplanes which is a normal crossing divisor. For a given $V \subset \mathbb{C}^{n}$, $\mathbb{P}^{r-1}=\mathbb{P} V$, and $H_{i}=\left\{x_{i}=0\right\}$. Hence, $\mathrm{KS}_{r, n}$ is the configuration space.

Kapranov defines his compactification $\overline{\mathrm{KS}}_{r, n}$ to be the Chow quotient $\operatorname{Gr}(r, n) / / T$ defined as follows. For any point $p \in \operatorname{Gr}^{0}(r, n)$, let the cycle $Z_{p}=\overline{T p}$ be the closure of the orbit of $p$ in $\operatorname{Gr}(r, n)$. This gives an embedding of $\mathrm{KS}_{r, n}$ into the Chow variety of $\operatorname{Gr}(r, n)$, and the latter is a projective variety. The compactification $\overline{\mathrm{KS}}_{r, n}$ is the closure of $\mathrm{KS}_{r, n}$ in this projective variety.

Definition 6.2 ([Kap93]). $\quad$ Let $G$ be an algebraic group acting on a projective variety $Y$ and let $Y^{0}$ be an open subset, assumed nonempty, where $G$ acts freely. The Chow quotient $Y / / G$ is the closure of $Y^{0} / G$ in the Chow variety of $Y$.

Alternatively, one can use the Plücker embedding

$$
\phi: \operatorname{Gr}(r, n) \rightarrow \mathbb{P}\left(\Lambda^{r} \mathbb{C}^{n}\right)
$$


Then for every $p \in \operatorname{Gr}^{0}(r, n)$ the closure of the orbit $\overline{T \phi(p)}$ is a torus-invariant cycle in $\mathbb{P}\left(\Lambda^{r} \mathbb{C}^{n}\right)$ and so gives a point of the Chow variety of $\mathbb{P}\left(\Lambda^{r} \mathbb{C}^{n}\right)$. Kapranov proves [Kap93] that one has an embedding of Chow quotients

$$
\overline{\mathrm{KS}}_{r, n}=\operatorname{Gr}(r, n) / / T \hookrightarrow \mathbb{P}\left(\Lambda^{r} \mathbb{C}^{n}\right) / / T
$$

The torus $T$ naturally acts on $\Lambda^{r} \mathbb{C}^{n}$ and the standard homogeneous coordinates of $\mathbb{P}\left(\Lambda^{r} \mathbb{C}^{n}\right)$ are eigenvectors. Therefore, they correspond to points in the character group of $T$, i.e., to integral points in $\mathbb{Z}^{n}$. Using the character group of $\left(\mathbb{C}^{*}\right)^{n}$ instead of the quotient torus $T$, these are the points that have $r$ coordinates equal to 1 and $(n-r)$ coordinates equal to 0 . The polytope with these $\left(\begin{array}{l}n \\ r\end{array}\right)$ vertices is called a hypersimplex and is denoted by $\Delta(r, n)$.

Example 6.3. $\Delta(1, n)$ is an ordinary $(n-1)$-dimensional simplex. $\Delta(2,4)$ is an octahedron. It is also obvious that $\Delta(r, n)$ and $\Delta(n-r, n)$ are isomorphic. Another way to describe $\Delta(r, n)$ is to say that its vertices are the barycenters of the $(r-1)$-dimensional faces of the $(n-1)$-dimensional simplex $r \Delta(1, n)$.

The advantage of using the second space is that the variety $\mathbb{P}\left(\Lambda^{r} \mathbb{C}^{n}\right) / / T$ is easily understood, see [KSZ91]. It is a projective, possibly non-normal toric variety $P_{\operatorname{Sec} \Delta(r, n)}^{\prime}$ whose normalization $\nu: P_{\operatorname{Sec} \Delta(r, n)} \rightarrow P_{\operatorname{Sec} \Delta(r, n)}^{\prime}$ corresponds to the secondary polytope $\operatorname{Sec} \Delta(r, n)$ of the hypersimplex.

To summarize, by [Kap93] one has

Theorem 6.4. There exists a natural injective map $\mathfrak{t}: \mathrm{KS}_{r, n} \rightarrow$ $P_{\mathrm{Sec} \Delta(r, n)}^{\prime}$ and one has $\overline{\mathrm{KS}}_{r, n}=\overline{\mathfrak{t}\left(\mathrm{KS}_{r, n}\right)}$.

Now, our basic observation is that both $\overline{\mathrm{KS}}_{r, n}$ and $P_{\mathrm{Sec} \Delta(r, n)}$ have functorial meaning as moduli spaces of stable pairs and that the morphism $\mathfrak{t}$ has meaning of a toric Torelli map.

Let us begin with the target space. There exist at least four related versions of it. Let $Q$ be an arbitrary maximal-dimensional lattice polytope in the lattice $\Lambda$ of characters of a torus $T$. Denote by $P_{Q}$ the projective toric variety associated to $Q$.

6.5. The original Chow quotient $\mathbb{P}^{N-1} / / T$, where $N=\#(\Lambda \cap Q)$. The canonical morphism $P_{Q} \rightarrow \mathbb{P}^{N-1}$ is finite. The points of the Chow quotient correspond to the cycle im $P_{Q}$, its translations $g$. im $P_{Q}, g \in\left(\mathbb{C}^{*}\right)^{N-1}$ under the action of the big diagonal torus of $\mathbb{P}^{N-1}$ and to limits of such cycles. The Chow quotient is a possibly non-normal toric variety $P_{\operatorname{Sec} Q}^{\prime}$ whose normalization is associated to the secondary polytope $\operatorname{Sec} Q$. 
It is well-known that the faces of $\operatorname{Sec} Q$ and hence the strata of $P_{\operatorname{Sec} Q}$ are in a bijection with convex lattice subdivisions of $Q$.

Advantages of the Chow quotient are its simplicity and its use of classical tools of projective geometry. Among the disadvantages, there are two. First, the procedure of taking a quotient provides only the structure of a reduced scheme. Secondly, the Chow variety does not represent any functor on the category of schemes. (One has to switch to a very restricted category of reduced weakly-normal schemes, see [Kol96]. Even then, this only works over a field, and the situation becomes quite complicated in positive characteristic.)

6.6. The toric Chow variety of [KSZ91]. The projective toric variety $P_{Q}$ comes with a finite morphism $P_{Q} \rightarrow \mathbb{P}^{N-1}$ which is dually described by a surjection $\pi: \sigma_{N} \rightarrow Q$ from the standard $(N-1)$-dimensional simplex $\sigma_{N}$. Fibers $\pi^{-1}(q)$ over points $q \in Q$ correspond to toric varieties $P_{q}$ (when $q$ is rational, $P_{q}$ comes with a natural ample $\mathbb{Q}$-line bundle). The polytope $Q$ is divided into finitely many polyhedral strata according to the types of the fibers. On the strata, there is a natural partial order by inclusion of the closures. One defines

$$
\operatorname{Chow}_{Q}=\lim P_{q}
$$

This is a projective scheme, possibly non-reduced and reducible. The main irreducible component of $\left(\mathrm{Chow}_{Q}\right)_{\text {red }}$ is a similar limit but in the category of $T$-varieties. The normalization of this variety is the projective toric variety $P_{\operatorname{Sec} Q}$ for the secondary polytope of $Q$.

The strata in the natural stratification of the toric Chow variety are in a bijection with all lattice subdivisions of $Q$.

This scheme does not represent a clearly defined moduli functor.

6.7. The toric Hilbert scheme of [PS02]. The toric Hilbert scheme parameterizes $\Lambda$-graded ideals $I$ in $R=k\left[x_{1}, \ldots, x_{N}\right]$ such that the graded pieces of the quotient $R / I$ are locally free of rank 0 or 1 . (There is a generalization to the case of arbitrary multiplicities, [HS02]).

The main component of toric Hilbert scheme corresponds to the state polytope with may be different from the secondary polytope.

6.8. The moduli space of stable toric pairs $M_{Q}$. This approach is used in [Ale02]. This is the coarse moduli space of stable toric varieties of numerical type $Q$. It also comes with a natural stratification in which strata are in a bijection with all lattice subdivisons of $Q$; and the normalization of the main irreducible component is $P_{\operatorname{Sec} Q}$. 
There exists a finite "Chow" morphism from $M_{Q}$ to the toric Chow variety with strata mapping to the strata with the same label.

A stable toric variety $P$ is a seminormal projective variety with a torus action so that there are only finitely many orbits and the stabilizers are connected and reduced. A stable toric pair $(P, D)$ adds an effective ample Cartier divisor not containing any orbits entirely. This is just a special case of the definition for a stable semiabelic pair we used in the previous Sections, when the semiabelian variety is a torus.

Let $(P, D)$ be a stable pair in which $P$ is a toric variety and with $(P, L=$ $\mathcal{O}(D))$ corresponding to a lattice polytope $Q \subset \Lambda_{\mathbb{R}}$. The equation $s \in H^{0}$ $(P, \mathcal{O}(D))$ written in the basis of $T$-eigenfuctions

$$
s=\sum_{m \in \Lambda \cap Q} c_{m} e_{m}
$$

can dually be interpreted as a finite map $\psi_{s}: P \rightarrow \mathbb{P}^{N-1}$, where $N=|\Lambda \cap Q|$ via the homomorphism

$$
\mathbb{C}\left[z_{1}, \ldots, z_{N}\right] \rightarrow \oplus_{d \geq 0} H^{0}\left(L^{d}\right), \quad z_{m} \mapsto c_{m} e_{m}
$$

This gives the connection with the Chow quotient: the data for the pair $(P,(s)=D)$ with toric $P$ is equivalent to the data for the cycle $\psi_{s}(P) \subset \mathbb{P}^{N-1}$.

6.9. Although in general the four moduli spaces are different, they all share a common subscheme $\operatorname{Unim}_{Q}$ corresponding to the unimodular convex subdivisions of $Q$. All the polyhedral decompositions appearing in the description of $\overline{\mathrm{KS}}_{r, n}$ are convex and unimodular, so all four approaches in this case are equivalent. We take the last approach, the moduli of stable pairs, as being just the finite version of the 2 nd Voronoi compactification $\overline{\mathrm{A}}_{g}^{\mathrm{Vor}}$ and the moduli space of stable pairs $\overline{\mathrm{AP}}_{g}$.

6.10. The source space.

Theorem 6.11. Points of $\overline{\mathrm{KS}}_{r, n} \backslash \mathrm{KS}_{r, n}$ correspond to pairs $(X, E)$ which are limits of pairs $\left(\mathbb{P}^{r-1}, \sum H_{i}\right)$. Moreover, the pairs $(X, E)$ are stable in the sense of [Ale96], i.e. the singularities are semi-log canonical and the sheaf $\omega_{X}(E)$ is ample.

We noticed this fact several years ago. Since the proof now appeared in a paper of Hacking [Hac03], we will omit it.

6.12. The analogy with the toroidal Torelli map is now complete. 


\section{§7. $\overline{\mathrm{KS}}_{2, n}$ Versus $\overline{\mathrm{M}}_{g}$}

We conclude with the following description of $\overline{\mathrm{K}}_{2, n}$ from [Kap93], which should be compared against the description of the compactified jacobians in 2.7.

\section{Theorem 7.1.}

(1) $\overline{\mathrm{KS}}_{2, n}$ coincides with $\overline{\mathrm{M}}_{0, n}$, the moduli space of $n$-pointed rational curves. It is naturally stratified by the types of curves, with strata labeled by trees $\Gamma$ with $n$ numbered ends.

(2) If $\left(X, E=P_{1}+\cdots+P_{n}\right)$ is in the stratum for the tree $\Gamma$ then $\overline{\mathfrak{t}}(X, E)$ is in the stratum corresponding to a decomposition of the polytope $\Delta(2, n)$ obtained by cutting it by hyperplanes.

(3) Hyperplanes correspond bijectively to the interior edges of $\Gamma$ (i.e. not the labeled end edges).

Problem 7.2. Give a moduli-theoretic description of the points of the stable pair $(P, D)$ corresponding to $\overline{\mathfrak{t}}(X, E)$ as semistable sheaves on $(X, E)$.

\section{Acknowledgements}

The first part of this paper was strongly influenced by the thought-provoking paper [OS79] of Oda and Seshadri. The second part is based on a talk at the September 2003 conference in honor of Prof. Tadao Oda's retirement, and it is a pleasure to dedicate this paper to him. My thanks also go to RIMS Kyoto University where this text was completed. The author was partially supported by NSF.

\section{References}

[AIK77] Altman, A. B., Iarrobino, A. and Kleiman, S. L., Irreducibility of the compactified Jacobian, Real and complex singularities (Proc. Ninth Nordic Summer School/NAVF Sympos. Math., Oslo, 1976), Sijthoff and Noordhoff, Alphen aan den Rijn, 1977, pp. 1-12.

[AK79] Altman, A. B. and Kleiman, S. L., Compactifying the Picard scheme. II, Amer. J. Math., 101 (1979), no. 1, 10-41.

[AK80] - Compactifying the Picard scheme, Adv. in Math., 35 (1980), no. 1, 50-112.

[Ale96] $\ldots$ Log canonical singularities and complete moduli of stable pairs, Preprint (1996), alg-geom/9608013.

[Ale02] Alexeev, V., Complete moduli in the presence of semiabelian group action, Ann. of Math. (2), 155 (2002), 611-708.

[Bea77] Beauville, A., Prym varieties and the Schottky problem, Invent. Math., 41 (1977), 149-196. 
[BM86] Beilinson, A. A. and Manin, Yu. I., The Mumford form and the Polyakov measure in string theory, Comm. Pure Appl. Math., 107 (1986), 359-376.

[Cap94] Caporaso, L., A compactification of the universal Picard variety over the moduli space of stable curves, J. Amer. Math. Soc., 7 (1994), 589-660.

[Cor89] Cornalba, M., Moduli of curves and theta-characteristics, Lectures on Riemann surfaces (Trieste, 1987), World Sci. Publishing, Teaneck, NJ, 1989, pp. 560-589.

[Cor91] , A remark on the Picard group of spin moduli space, Rend. Mat. Acc. Lincei, 2 (1991), 211-217.

[DH98] Dolgachev, I. V. and Hu, Y., Variation of geometric invariant theory quotients, Inst. Hautes Études Sci. Publ. Math., 87 (1998), 5-56, With an appendix by Nicolas Ressayre.

[D'S79] D'Souza, C., Compactification of generalised Jacobians, Proc. Indian Acad. Sci. Sect. A Math. Sci., 88 (1979), no. 5, 419-457.

[Est95] Esteves, E., Three times the theta divisor on the compactified jacobian is very ample, Preprint (1995).

[Hac03] Hacking, P., Compact moduli of hyperplane arrangements, math. AG/0310479.

[HS02] Haiman, M. and Sturmfels, B., Multigraded Hilbert schemes, math. AG/0201271.

[Kap93] Kapranov, M. M., Chow quotients of Grassmannians. I, I. M. Gel'fand Seminar, Adv. Soviet Math., vol. 16, Amer. Math. Soc., Providence, RI, 1993, pp. 29-110.

[KK81] Kleppe, H. and Kleiman, S. L., Reducibility of the compactified Jacobian, Compositio Math., 43 (1981), 277-280.

[KM76] Knudsen, F. and Mumford, D., The projectivity of the moduli space of stable curves I, Math. Scand., 39 (1976), 19-55.

[Kol96] Kollár, J., Rational curves on algebraic varieties, Ergeb. Math. Grenzgeb. (3), 32, Springer-Verlag, Berlin, 1996.

[KSZ91] Kapranov, M. M., Sturmfels, B. and Zelevinsky, A. V., Quotients of toric varieties, Math. Ann., 290 (1991), 643-655.

[Laf03] Lafforgue, L., Chirurgie des grassmanniennes, CRM Monograph Series, vol. 19, American Mathematical Society, Providence, RI, 2003.

[Nam76] Namikawa, Y., A new compactification of the Siegel space and degenerations of abelian varieties, I, II., Math. Ann., 221 (1976), 97-141, 201-241.

[OS79] Oda, T. and Seshadri, C. S., Compactifications of the generalized jacobian variety, Trans. Amer. Math. Soc., 253 (1979), 1-90.

[Ox192] Oxley, J. G., Matroid theory, Oxford University Press, 1992.

[PS02] Peeva, I. and Stillman, M., Toric Hilbert schemes, Duke Math. J., 111 (2002), 419-449.

[Ses82] Seshadri, C. S., Fibrés vectoriels sur les courbes algébriques, Astérisque, 96 (1982).

[Sim94] Simpson, C. T., Moduli of representations of the fundamental group of a smooth projective variety I, Inst. Hautes Études Sci. Publ. Math. (1994), no. 79, 47-129.

[Sou94] Soucaris, A., The ampleness of the theta divisor on compactified jacobian of a proper and integral curve, Compositio Math., 93 (1994), no. 231-242.

[Vor09] Voronoi, G., Nouvelles applications des paramètres continus à la théorie des formes quadratique, I, II, III, J. Reine Angew. Math., 133,134,136 (1908,1909), 97-178, 198-287, 67-181.

Note added in proof: Alternative proof of seminormality of $R / /$ PGL in Thm. 5.1: $R \rightarrow R / /$ PGL factors uniquely through $f: R \rightarrow Z$ by a universal property of seminormali zation. By uniqueness, $f$ is PGL-equivariant. But then $f$ factors through the categorial quotient $R / / \mathrm{PGL}$ and $Z=R / / P G L$. 